\title{
Application of a MALDI-TOF analysis platform (ClinProTools) for rapid and preliminary report of MRSA sequence types in Taiwan
}

\author{
Hsin-Yao Wang ${ }^{1,2}$ ， Tsui-Ping Liu ${ }^{1}$ ， Frank Lien ${ }^{1}$ ， Chun-Hsien Chen ${ }^{3}$ ， Chao-Jung Chen ${ }^{\text {Corresp., }} 4,5$, Jang-Jih Lu \\ Corresp. 1, 6, 7 \\ 1 Department of Laboratory Medicine, Chang Gung Memorial Hospital at Linkou, Tauyuan, Taiwan \\ 3 Department of Information Management, Chang Gung University, Taoyuan, Taiwan \\ 4 Graduate Institute of Integrated Medicine, China Medical University, Taichung, Taiwan \\ 5 Proteomics Core Laboratory, China Medical University Hospital, Taichung, Taiwan \\ 6 Department of Medical Biotechnology and Laboratory Science, Chang Gung University, Taoyuan, Taiwan \\ 7 School of Medicine, Chang Gung University, Taoyuan, Taiwan \\ Corresponding Authors: Chao-Jung Chen, Jang-Jih Lu \\ Email address: cjchen@mail.cmu.edu.tw, jjlpcp@cgmh.org.tw
}

\section{Background}

The accurate and rapid preliminarily identification of the types of methicillin-resistant Staphylococcus aureus (MRSA) is crucial for infection control. Currently, however, expensive, time-consuming, and laborintensive methods are used for MRSA typing. By contrast, matrix-assisted laser desorption ionization time-of-flight mass spectrometry (MALDI-TOF MS) is a potential tool for preliminary lineage typing. The approach has not been standardized, and its performance has not been analyzed in some regions with geographic barriers (e.g., Taiwan island).

\section{Methods}

The mass spectra of 306 MRSA isolates were obtained from multiple reference hospitals in Taiwan. The multilocus sequence typing (MLST) types of the isolates were determined. The spectra were analyzed for the selection of characteristic peaks by using the ClinProTools software. Furthermore, various machine learning (ML) algorithms were used to generate binary and multiclass models for classifying the major MLST types (ST5, ST59, and ST239) of MRSA.

\section{Results}

Ten peaks with the highest discriminatory power (m/z range: 2082-6594) were identified and evaluated. All the single peaks revealed significant discriminatory power during MLST typing. Moreover, the binary and multiclass ML models achieved sufficient accuracy $(82.80 \%-94.40 \%$ for binary models and $>81.00 \%$ for multiclass models) in classifying the major MLST types.

\section{Conclusions}

A combination of MALDI-TOF MS analysis and ML models is a potentially accurate, objective, and efficient tool for infection control and outbreak investigation. 


\section{Application of a MALDI-TOF Analysis Platform}

\section{2 (ClinProTools) for Rapid and Preliminary Report of MRSA}

\section{Sequence Types in Taiwan}

4 Hsin-Yao Wang ${ }^{\dagger 1,2}$, Tsui-Ping Liu ${ }^{\dagger 1}$, Frank Lien ${ }^{\dagger 1}$, Chun-Hsien Chen ${ }^{3}$, Chao-Jung Chen ${ }^{* 4,5}$, Jang-Jih

$5 \mathrm{Lu}^{* 1,6,7}$

$6{ }^{1}$ Department of Laboratory Medicine, Chang Gung Memorial Hospital at Linkou, Taoyuan City, Taiwan

$7 \quad{ }^{2}$ Ph.D. Program in Biomedical Engineering, Chang Gung University, Taoyuan City, Taiwan

$8{ }^{3}$ Department of Information Management, Chang Gung University, Taoyuan City, Taiwan

$9{ }^{4}$ Graduate Institute of Integrated Medicine, China Medical University, Taichung City, Taiwan

105 Proteomics Core Laboratory, China Medical University Hospital, Taichung City, Taiwan

$11{ }^{6}$ Department of Medical Biotechnology and Laboratory Science, Chang Gung University, Taoyuan City,

12 Taiwan

$13{ }^{7}$ School of Medicine, Chang Gung University, Taoyuan City, Taiwan

14

$15 \dagger$ Contributed equally to this work

$16 *$ Corresponding Author:

17 1. Jang-Jih Lu

18 No. 5, Fuxing Street, Guishan District, Taoyuan City, Taiwan

19 Email address: jjlpcp@cgmh.org.tw

20 2. Chao-Jung Chen

21 No. 2, Yude Road, North District, Taichung City, Taiwan

22 Email address: cjchen@mail.cmu.edu.tw 


\section{Abstract}

\section{Background}

25 The accurate and rapid preliminarily identification of the types of methicillin-resistant 26 Staphylococcus aureus (MRSA) is crucial for infection control. Currently, however, expensive, 27 time-consuming, and labor-intensive methods are used for MRSA typing. By contrast, matrix28 assisted laser desorption ionization time-of-flight mass spectrometry (MALDI-TOF MS) is a 29 potential tool for preliminary lineage typing. The approach has not been standardized, and its 30 performance has not been analyzed in some regions with geographic barriers (e.g., Taiwan 31 island).

\section{Methods}

33 The mass spectra of 306 MRSA isolates were obtained from multiple reference hospitals in

34 Taiwan. The multilocus sequence typing (MLST) types of the isolates were determined. The 35 spectra were analyzed for the selection of characteristic peaks by using the ClinProTools 36 software. Furthermore, various machine learning (ML) algorithms were used to generate binary 37 and multiclass models for classifying the major MLST types (ST5, ST59, and ST239) of MRSA.

\section{Results}

39 Ten peaks with the highest discriminatory power (m/z range: 2082-6594) were identified and 40 evaluated. All the single peaks revealed significant discriminatory power during MLST typing.

41 Moreover, the binary and multiclass ML models achieved sufficient accuracy $(82.80 \%-94.40 \%$ 42 for binary models and $>81.00 \%$ for multiclass models) in classifying the major MLST types.

\section{Conclusions}

44 A combination of MALDI-TOF MS analysis and ML models is a potentially accurate, objective, 45 and efficient tool for infection control and outbreak investigation. 


\section{Introduction}

47 Since their emergence in the 1960s, methicillin-resistant Staphylococcus aureus (MRSA)

48 infections have been a major health care concern worldwide (Chen \& Huang 2014; Walter et al.

49 2015; Wang et al. 2010). Many epidemiological studies have revealed that different multilocus

50 sequence types (MLST) present specific characteristics such as virulence gene profiles (Recker

51 et al. 2017; Schuenck et al. 2012; Wang et al. 2010; Wang et al. 2012; Wang et al. 2009).

52 Understanding the evolution of MRSA lineages and the origin of infection is crucial in outbreak

53 investigation. Molecular typing methods, such as pulsed-field gel electrophoresis and MLST, are

54 highly expensive and labor intensive for epidemiological studies. Hence, the application of these

55 methods in clinical practice is limited (Struelens et al. 2009). Sequence based typing methods has

56 been widely used since the past decade, and it provides adequately high resolution for

57 confirming transmission. However, in regions with few medical resources and financial

58 constraints, it remains relatively impracticable. (Harris et al. 2013; Koser et al. 2012; Schwarze

59 et al. 2018)

60 Recently, matrix-assisted laser desorption time-of-flight mass spectrometry (MALDI-TOF

61 MS) has been used in many clinical microbiology laboratories. This method can be used to

62 identify bacterial species effectively and rapidly (Ge et al. 2016). The peptide or protein MS

63 fingerprint of each bacterium can be generated and stored as a in a bacterial library for species

64 identification. In addition, MALDI-TOF MS also provides an alternative solution for molecular

65 typing methods (e.g., MLST) (Lartigue 2013; Lu et al. 2012) and has the potential to offer

66 lineage typing up to the subspecies level.

67 Studies that have adopted the MALDI-TOF MS approach (Lasch et al. 2014; Sauget et al.

68 2017; Ueda et al. 2015) have reported varying results; it may be due to several reasons. First, the 
69 predominant MRSA lineages in different areas are different and the discriminatory power of

70 MALDI-TOF might therefore differ when compared between different MRSA lineages. Second,

71 the bacterial MS fingerprints of isolates from one area may not match those of isolates from 72 other areas. Third, in many of the published works, the MALDI-TOF mass spectra have been 73 assessed manually. An objective, standardized, and automated protocol has not been widely 74 applied thus far (Camoez et al. 2016). Finally, the data obtained from MALDI-TOF mass spectra 75 are relatively complicated and may be analyzed by a wide variety of bioinformatics tools. A 76 manual approach cannot ensure a consistently high-quality output, because of the potential for 77 large interindividual or intraindividual variation in the interpretation of the data. Therefore, a 78 reliable analysis platform is necessary to ensure comparability between reports in clinical 79 practice. Although Staphylococcus protein A (spa) typing provides comparable performance as 80 MLST typing with less cost and time, our study has adopted MLST typing because we aim to 81 demonstrate the possibility to implement our method beyond S. aureus. (Crisostomo et al. 2001; 82 O'Hara et al. 2016) To validate the use of MALDI-TOF mass spectra in classifying MLST types 83 of MRSA in Taiwan, we used the ClinProTools software for analyzing MALDI-TOF mass 84 spectra to generate classification models of MRSA lineages. Accordingly, clinical microbiology 85 laboratories may rapidly provide preliminary typing reports of MRSA, which may be further 86 confirmed using a sequence-based method, thus enabling clinical practitioners to exclude an 87 outbreak or transmission in time.

\section{Materials \& Methods}

\section{Bacterial lineages}

91 This study included 306 convenience non-duplicate MRSA lineages isolated from multiple 
92 reference hospitals in Taiwan, mainly through the Surveillance of Multicenter Antimicrobial

93 Resistance in Taiwan (SMART) program (Wang et al. 2012). The SMART program

94 consecutively collected MRSA isolates from ten medical centers throughout Taiwan from March

95 to August 2003. (Ho et al. 2010) All the lineages in this study were convenience samples which

96 had been recovered from blood cultures. All duplicate isolates were removed from the study.

97 After procuring the cultures from bacterial banks, tests were performed again to confirm the

98 characteristics of each lineage. The identification of S. aureus was based on colony morphology,

99 microscopic examination, a coagulase test, a catalase test, and MALDI-TOF mass spectra.

100

101

102

103

105

106

107

108

109

110

111

112

113

114

\section{Bacterial identification through MALDI-TOF MS}

The fresh bacterial colonies that were grown on blood agar plates for 24 hours were picked up and smeared onto a MALDI steel target plate, forming a thin film of colonies. Next, $1 \mu \mathrm{L}$ of $70 \%$ formic acid was introduced on the film and dried at room temperature. Subsequently, $1 \mu \mathrm{L}$ of the matrix solution (i.e., $50 \%$ acetonitrile containing 1\% $\alpha$-cyano-4-hydroxycinnamic acid and $2.5 \%$ trifluoroacetic acid) was introduced on the film again. The sample-matrix was dried at room temperature before analyzing it through MS for data acquisition. Mass spectrum analysis was performed using a MicroFlex LT mass spectrometer (Bruker Daltonik GmbH, Bremen, Germany) with linear positive model, and the analytic region was 2000-20000 Da. For each sample, 240 laser shots (at frequency of $20 \mathrm{~Hz}$ ) were collected, and a Bruker Daltonics Bacterial test standard (Bruker Daltonik $\mathrm{GmbH}$ ) was used for calibration and as the control with the linear positive model. The procedures were conducted according to the manufacturer's instructions, which have been detailed in previous studies (Ge et al. 2016; Lu et al. 2012). The results of mass spectrum from the MALDI Biotyper 3.1 software (Bruker Daltonik GmbH) were compared with 
115 those in the database and assigned scores. Peaks with scores $>2$ were further selected for peak

116 signal analysis. The lineages were randomly divided into batches, and the analyses were

117 conducted on different days to avoid a possible batch effect.

\section{MLST}

120 We sequenced the lineages for seven housekeeping genes, namely carbamate kinase $(\operatorname{arc} C)$, 121 shikimate dehydrogenase (aroE), glycerol kinase $(g l p F)$, guanylate kinase (gmk), phosphate 122 acetyltransferase ( $p t a)$, triosephosphateisomerase (tpi), and acetyl coenzyme A acetyltransferase 123 (yqiL). The sequencing results of these genes were compared with those in the $S$. aureus MLST 124 database (http://saureus.mlst.net/) to acquire an allelic number and a sequence type (Enright et al. 125 2000).

\section{MALDI-TOF MS Spectra Analysis}

MALDI-TOF mass spectra of the MRSA lineages were fed into the ClinProTools ${ }^{\mathrm{TM}}$ 129 software (version 3.0, Bruker Daltonik $\mathrm{GmbH}$ ) in batches. The data preprocessing steps, including baseline subtraction, smoothing, and recalibration, were set as default for all analyses (Bruker Daltonik GmbH 2011; Camoez et al. 2016; Zhang et al. 2015). ClinProTools is a widely used software developed by Bruker (Bruker Daltonik GmbH). It has been used in MALDI-TOF data analysis for MRSA lineages typing (Camoez et al. 2016; Zhang et al. 2015). Characteristic

134 peaks among various MLST types were selected and sorted through several statistical tests, 135 including the t-test, analysis of variance (ANOVA), the Wilcoxon or Kruskal-Wallis (W/KW) 136 test, and the Anderson-Darling (AD) test. A $P$ value of 0.05 was set as the cutoff. If $P$ was $<0.05$ 137 in the AD test, a characteristic peak was selected if the corresponding value of $P$ in the $\mathrm{W} / \mathrm{KW}$ 
138 test was also $<0.05$. When $P$ was $\geq 0.05$ in the AD test, then a characteristic peak was selected if

139 the corresponding value of $P$ in ANOVA was also $<0.05$ (Stephens 1974).

140

\section{Generation and validation of classification models}

142

143

144

145

146

147

148

149

150

151

152

153

154

155

156

\section{Statistical analysis}

158

159

160

Classification models of the major MLST types (ST5, ST59, and ST239) were generated using the ML algorithms in ClinProTools, namely QuickClassifier (QC), Supervised Neural Network (SNN), and Genetic Algorithm-K Nearest Neighbor (GA-KNN). The description and setting of the ML models are detailed in the ClinProTools user manual (Bruker Daltonik GmbH 2011). All the peaks in the spectra were used in model generation. The $\mathrm{W} / \mathrm{KW}$ test was used to sort peaks during selection. For GA-KNN, GA was used as the method for feature selection, where the maximum number of best peaks was set as 30, and the maximum number of generations was set as 50. The numbers of nearest neighbors evaluated in the GA-KNN algorithm were 1, 3, and 5 to 7 for each binary classification. To avoid overfitting, we used 5fold cross validation to obtain an unbiased statistical measurement of performance. Accordingly, the data were split into five subsets in a randomized manner. Each subset would serve as the validation set for the model trained by the remaining four subsets iteratively. Classification accuracy was obtained from the average of the five evaluations. Consequently, the bias of overfitting could be avoided using 5-fold cross validation.

The AD test is used to test for a normal distribution of peak intensity in ClinProTools.

In the $\mathrm{AD}$ test when $P \leq 0.05$ (i.e., the data distribution did not follow normal distribution), the $\mathrm{W} / \mathrm{KW}$ test was used as the statistical method to select discriminative peaks. In the $\mathrm{W} / \mathrm{KW}$ 
161 test, if $P \leq 0.05$, a rank-based multiple test procedure was used for post-hoc analysis to conduct

162 paired comparisons in the nonnormal distributed data and calculate the simultaneous confidence

163 intervals with Tukey-type contrasts (Konietschke et al. 2012). For evaluating the performance of

164 various ML models, accuracy, sensitivity, and specificity were used as the metrics.

165

166

167

168

169

170

171

172

173

174

175

176

177

178

179

180

181

182

183

$$
\text { Accuracy }=\frac{T P+T N}{T P+F P+T N+F N}
$$

$$
\text { Sensitivity }=\frac{T P}{T P+F N}
$$$$
\text { Specificity }=\frac{T N}{T N+F P}
$$

where TP, TN, FP, and FN represent the number of true positives, true negatives, false positives, and false negatives, respectively.

\section{Results}

Six MLST types of MRSA were identified in the isolates, namely ST5 $(\mathrm{n}=40)$, ST45 $(\mathrm{n}=$ 8), ST59 $(n=62), \operatorname{ST} 239(n=179)$, ST241 $(n=12)$, and ST573 $(n=5)$. The isolates ST5, ST59, and ST239 were considered the major MLST types of MRSA because they exhibited numerous lineages. The isolates ST45, ST241, and ST573 were categorized together as other ST types.

\section{Characteristic peaks for discrimination among various MLST types}

The characteristic peaks were sorted using the corresponding $P$ values obtained in the $(\mathrm{W} / \mathrm{KW}$ ) test because the AD test revealed $P<0.05$ (Table 1). The top 10 characteristic peaks, sorted using the W/KW test results, were selected for further statistical evaluation. The 10 peaks ranged from $m / z 2082-6594$ (Table 1). All the 10 selected peaks revealed $P<0.000001$ in the $\mathrm{W} / \mathrm{KW}$ test, thus indicating that they were informative and discriminative peaks in the MLST type classification.

In Table 1, the distribution of these peaks over various MLST types was further evaluated. 
184 High expression levels of $m / z 2430$ and 3893 and a low expression level of $\mathrm{m} / \mathrm{z} 3877$ indicated 185 the fingerprint of ST5; low expression levels of $m / z 2416,2980$, and 3893 indicated the 186 fingerprint of ST59; high expression levels of $\mathrm{m} / \mathrm{z} 2416$ and 2880 and low expression levels of $187 \mathrm{~m} / \mathrm{z} 3277$ and 3893 indicated the fingerprint of ST239. Based on 10 informative peaks, the odds 188 ratio $(\mathrm{OR})$ of different ST-pairs was calculated to determine the association between the peaks 189 and respective ST-pairs (Supplemental Table S1). Furthermore, the distribution of the isolates 190 was plotted according to peak intensity of $m / z 3277$ (x axis) and $m / z 6594$ (y axis) (Fig. 1). The 191 peaks at $m / z 3277$ and 6594 were the top two characteristic peaks among the ST types (Fig. 1).

192 The scatter plot figures may be seen as a projection of a high-dimensional scatter space with 193 various dimensions of the $\mathrm{m} / \mathrm{z}$ peaks.

194 The average intensities of the 10 peaks of these lineages are further illustrated in Fig. 2. 195 These results demonstrate the specific characteristics and patterns of peaks in the different 196 MLST types. Specifically, the distribution of the ST59 lineages and ST239 lineages showed 197 satisfactory separation (Fig. 1); ST5 lineages could also be distinguished from ST239 lineages 198 based on distribution (Fig. 1). By contrast, ST5 lineages and ST59 lineages could not be 199 satisfactorily discriminated using information from the peaks $m / z 3277$ and 6594 only (Fig. 1). 200 Similarly, the lineages of other minor ST types mixed with other major ST types (i.e., ST5, ST59, 201 and ST239) on the scatter plot (Fig. 1).

202

\section{ML models for classification of various MLST types of MRSA}

Various MLST types showed specific patterns of peaks expression, as presented in Table 1 205 and Fig. 3-5. To generate a comprehensive and objective classification, ML algorithms were used. In the ST5 binary classification models, the peaks at $m / z 3877$ and $\mathrm{m} / \mathrm{z} 3893$ showed 
207 satisfactory discriminative power (Fig. 3). The QC algorithm attained the highest cross208 validation values $(94.40 \%$, Table $2(\mathrm{a}))$.

209 In ST59 binary classification models, peaks at $\mathrm{m} / \mathrm{z} 2980$ and $\mathrm{m} / \mathrm{z} 2416$ also showed 210 satisfactory discriminative power (Fig. 4). The GA-KNN algorithm attained the highest cross211 validation values among all the algorithms. The GA-KNN algorithm showed highest

212 performance when the number of nearest neighbor was set as $5(85.00 \%$, Table 2(b)).

213 For the binary classification of ST239 versus non-ST239, the peaks at $\mathrm{m} / \mathrm{z} 3277$ and $\mathrm{m} / \mathrm{z}$ 2146553 showed moderate discriminative power (Fig. 5). The GA-KNN algorithm showed higher 215 performance over other algorithms when the number of nearest neighbor was set as $7(82.80 \%$, 216 Table 2(c)).

217 In this study, multiclass models for classifying an isolate into one of four lineages were 218 designed. Generally, the GA-KNN algorithm outperformed the other algorithms; more 219 specifically, this algorithm was suitable for detecting ST239 (Table 3). The GA-KNN algorithm 220 could successfully detect the ST5, ST59, and ST239 lineages with an accuracy of $>81.00 \%$. 221 However, none of the multiclass models showed reliable accuracy in detecting isolates of the 222 other ST lineages (i.e., ST45, ST241, and ST573).

223

\section{Discussion}

225 In this study, the major ST types (i.e., ST5, ST59, and ST239) of blood stream MRSA in 226 Taiwan could be classified through ML-based MALDI-TOF mass spectra analysis with high 227 accuracy. The analysis was conducted using a standardized system (i.e., ClinProTools) to obtain 228 objective and consistent results. Moreover, the ST types of MRSA could be predicted accurately 229 through MALDI-TOF mass spectra analysis before additional molecular typing methods (e.g., 230 MLST). Biomarker peaks of various MRSA ST types were discovered through MALDI-TOF 
231 mass spectra analysis by using the ClinProTools software. Some of the biomarker peaks have

232 been previously reported (Camoez et al. 2016; Josten et al. 2013; Sauget et al. 2017; Zhang et al.

2332015 ) and validated in the study (namely $m / z 3277,3877,3893,6553$, and 6594), whereas some

234 of them have not yet been widely validated (namely $m / z$ 2082, 2416, 2430, 2880, and 2980).

235 Nevertheless, reports on the MS characteristics of MRSA are inconsistent (Lasch et al. 2014;

236 Sauget et al. 2017; Ueda et al. 2015). Although the accuracy of typing is sufficient in individual

237 studies, the general patterns of specific lineages are not available (Sauget et al. 2017). Currently,

238 bacterial lineages obtained from geographically diverse areas cannot be clearly discriminated

239 using MALDI-TOF MS. However, more robust characteristic patterns of various types may be

240 available in regions with geographic barriers (e.g., Taiwan island) than in regions without these

241 barriers. The characteristic patterns of specific lineages may be the result of the disseminated

242 lineages in local areas. By contrast, establishing a localized solution by using an appropriate

243 method of interpreting mass spectra may be more practical and crucial than establishing a

244 generalized pattern. The isolates in this study were obtained from multiple reference hospitals in

245 Taiwan. Consequently, the isolates used in the study can represent the molecular characteristics

246 of MRSA in the local region. The localized molecular characteristics of MRSA may be

247 sufficiently useful for clinical practice in regions with geographic barriers (e.g., Taiwan island).

248 Currently, clinical microbiology laboratories generally use MALDI-TOF MS for bacterial

249 identification because of its advantages in accuracy and effectiveness over traditional

250 biochemical methods (Lartigue 2013). Before analytical measurement by using MALDI-TOF

$251 \mathrm{MS}$, a protein extraction process is necessary. In-tube extraction or the direct deposit method are

252 two common extraction methods used in clinical microbiology laboratories. In-tube extraction

253 provides more purified intracellular components than the direct deposit method does, which

254 results in high quality MALDI-TOF mass spectra and low noise. By contrast, the direct 
255 deposition of bacteria onto a steel plate is considered a less labor-intensive and more rapid 256 preanalytical process than in-tube extraction. The time for the entire process and the turnaround

257 time of MALDI-TOF MS can be considerably reduced by using the direct deposit method. 258 Consequently, considering the relevance in routine practice, the direct deposit method was 259 evaluated in this study.

260 Knowledge of the bacterial molecular type of MRSA is crucial while performing 261 epidemiological studies on bacterial outbreak. In this study, specific peaks were identified for 262 major clonal lineages of MRSA. The peaks $\mathrm{m} / \mathrm{z}$ near 3277, 3877, 3893, 6553, and 6594 identified 263 in this study have been reported in previous studies as characteristic peaks in discriminating 264 MRSA clonal complexes (CCs). (Camoez et al. 2016; Josten et al. 2013; Wolters et al. 2011). 265 Zhang et al. reported the highest expression of the peaks at $m / z 3277$ and 6554 in ST59 (Zhang et 266 al. 2015). The peak at $m / z 6594$ was recognized as the SA1452 protein and as a biomarker of the 267 clonal complex $8(\mathrm{CC} 8)$ and USA-300 lineages; both these MRSA lineages are prevalent in 268 communities and hospitals (Boggs et al. 2012; Josten et al. 2013; Wolters et al. 2011). In this 269 study, the peak at $\mathrm{m} / z 6594$ was characteristic for ST239, which were the most prominent ST 270 type of bloodstream MRSA and prominent HA MRSA (Table 1). By contrast, some of the 271 characteristic peaks have not previously been reported and validated as discriminative peaks (i.e., 272 peaks at $m / z$ 2082, 2416, 2430, 2880, and 2980) (Sauget et al. 2017); however, they play a 273 crucial role in MLST type classification. For example, the peak at $m / z 2980$ was a distinguishing 274 peak for the ST59 MRSA lineages (Fig. 4), which is one of the characteristic ST types of the CA 275 MRSA in Taiwan (Huang \& Chen 2011). Moreover, the peaks at $\mathrm{m} / \mathrm{z} 2416$ and 2430 were noted 276 as characteristic peaks in the ST5 and ST239. In the QC models, the peak at $m / z 2416$ was 277 proven to represent psm-mec, which is strongly associated with SCCmec III and VIII (Queck et 278 al. 2009). Briefly, although the peak at $m / z 2416$ is commonly expressed peak in MRSA, the 
279 expression level, in addition to its presence, may serve as an informative feature in classification 280 of MRSA lineages.

281 Furthermore, for identifying subtle differences in the MALDI-TOF mass spectra for 282 preliminary reporting of AST or subspecies results, not only single characteristic peaks but also 283 specific combinations of characteristic peaks may be beneficial. Single MS peaks have provided 284 some characteristics of each major ST type (Table 1, Fig. 1 to Fig. 5). Additional integration of 285 these characteristic peaks by using ML models may generate a more comprehensive and robust 286 result for ST typing of MRSA (Table 2) than that generated using single peaks. Consequently, 287 the accuracy of the subspecies classification by using a combination of peaks may be higher and more resistant to variations in analysis than that using a single peak because of comprehensive interpretation. Zhang et al. reported the successful use of ML models (by ClinProTools) in analyzing MALDI-TOF mass spectra to classify various MLST types of MRSA (Zhang et al. 2015). High performance of the binary models (including ST5, ST45, ST59, and ST239 binary classification models) were described. However, the high performance might have resulted from overfitting because only one specific combination of training sets and validation sets was used 294 for performance evaluation.

295 This study had several limitations. Firstly, our study did not adopt nucA PCR or other 296 sequencing methods to exclude $S$. argenteus, which were previously identified as $S$. aureus using molecular typing (Thaipadungpanit et al. 2015; Tong et al. 2015). The MRSA isolates were 298 collected from an island with geographic barriers, thus resulting in a relative simple ST 299 distribution. Possibly, our method might be unsuitable in other country with wide variety of 300 bacterial lineage. We thus recommend researchers who intend to adopt our approach to train and 301 validate their own model by using regional MRSA lineages. Another limitation of this study is 302 that the MRSA isolates in this study are all from previously collected samples under the SMART 
303 program. This indicates the model may not exhibit sufficient accuracy in performance on new

304 MRSA isolated in the future. Moreover, only six MLST types (namely ST5, ST45, ST59, ST239,

305 ST241 and ST573) of MRSA were included for models training and validation. Based on the

306 study design, ML learning models can be used for detecting the major lineages of MRSA in

307 Taiwan only, but they cannot be used for detecting new clones, which were not included in

308 model training. Consequently, the models are designed to be used for reporting preliminary

309 lineage information in outbreak investigation. When a new clone emerges in the future, the ML

310 models could be tuned and updated using the newly collected datasets. Our study has

311 demonstrated that in regions with limited medical resources, an MALDI-TOF-based MLST

312 typing model may serve as a valuable method for timely intervention in the transmission or

313 outbreak of MRSA.

314

315 Acknowledgments

316 This manuscript was edited by Wallace Academic Editing.

317

318 References

319 Boggs SR, Cazares LH, and Drake R. 2012. Characterization of a Staphylococcus aureus USA300

320

321 protein signature using matrix-assisted laser desorption/ionization time-of-flight mass spectrometry. J Med Microbiol 61:640-644. 10.1099/jmm.0.037978-0

322

323

324

325

326

327

328

329

Chen CJ, and Huang YC. 2014. New epidemiology of Staphylococcus aureus infection in Asia. Clin Microbiol Infect 20:605-623. 10.1111/1469-0691.12705 
330

331

332

333

334

335

336

337

338

339

340

341

342

343

344

345

346

347

348

349

350

351

352

353

354

355

356

357

358

359

360

361

362

363

364

365

Crisostomo MI, Westh H, Tomasz A, Chung M, Oliveira DC, and de Lencastre H. 2001. The evolution of methicillin resistance in Staphylococcus aureus: similarity of genetic backgrounds in historically early methicillin-susceptible and -resistant isolates and contemporary epidemic clones. Proc Natl Acad Sci U S A 98:9865-9870. 10.1073/pnas.161272898

Enright MC, Day NP, Davies CE, Peacock SJ, and Spratt BG. 2000. Multilocus sequence typing for characterization of methicillin-resistant and methicillin-susceptible clones ofStaphylococcus aureus. J Clin Microbiol 38:1008-1015.

Ge MC, Kuo AJ, Liu KL, Wen YH, Chia JH, Chang PY, Lee MH, Wu TL, Chang SC, and Lu JJ. 2016. Routine identification of microorganisms by matrix-assisted laser desorption ionization time-of-flight mass spectrometry: Success rate, economic analysis, and clinical outcome. J Microbiol Immunol Infect. 10.1016/j.jmii.2016.06.002

Harris SR, Cartwright EJP, Török ME, Holden MTG, Brown NM, Ogilvy-Stuart AL, Ellington MJ, Quail MA, Bentley SD, Parkhill J, and Peacock SJ. 2013. Whole-genome sequencing for analysis of an outbreak of meticillin-resistant Staphylococcus aureus: a descriptive study. The Lancet Infectious Diseases 13:130-136. 10.1016/s1473-3099(12)70268-2

Ho CM, Hsueh PR, Liu CY, Lee SY, Chiueh TS, Shyr JM, Tsao SM, Chuang YC, Yan JJ, Wang LS, Wang JH, Ho MW, Tien N, and Lu JJ. 2010. Prevalence and accessory gene regulator (agr) analysis of vancomycin-intermediate Staphylococcus aureus among methicillin-resistant isolates in Taiwan--SMART program, 2003. Eur J Clin Microbiol Infect Dis 29:383-389. 10.1007/s10096-009-0868-4

Huang YC, and Chen CJ. 2011. Community-associated meticillin-resistant Staphylococcus aureus in children in Taiwan, 2000s. Int $J$ Antimicrob Agents 38:2-8. 10.1016/j.ijantimicag.2011.01.011

Josten M, Reif M, Szekat C, Al-Sabti N, Roemer T, Sparbier K, Kostrzewa M, Rohde H, Sahl HG, and Bierbaum G. 2013. Analysis of the Matrix-Assisted Laser Desorption Ionization-Time of Flight Mass Spectrum of Staphylococcus aureus Identifies Mutations That Allow Differentiation of the Main Clonal Lineages. J Clin Microbiol 51:1809-1817. 10.1128/jcm.00518-13

Konietschke F, Hothorn LA, and Brunner E. 2012. Rank-based multiple test procedures and simultaneous confidence intervals. Electronic Journal of Statistics 6:738-759. 10.1214/12-EJS691

Koser CU, Holden MT, Ellington MJ, Cartwright EJ, Brown NM, Ogilvy-Stuart AL, Hsu LY, Chewapreecha C, Croucher NJ, Harris SR, Sanders M, Enright MC, Dougan G, Bentley SD, Parkhill J, Fraser L, Betley JR, Schulz-Trieglaff OB, Smith GP, and Peacock SJ. 2012. Rapid whole-genome sequencing for investigation of a neonatal MRSA outbreak. $N$ Engl J Med 
366

367

368

369

370

371

372

373

374

375

376

377

378

379

380

381

382

383

384

385

386

387

388

389

390

391

392

393

394

395

396

397

398

399

400

401

366:2267-2275. 10.1056/NEJMoa1109910

Lartigue MF. 2013. Matrix-assisted laser desorption ionization time-of-flight mass spectrometry for bacterial strain characterization. Infect Genet Evol 13:230-235. 10.1016/j.meegid.2012.10.012

Lasch P, Fleige C, Stämmler M, Layer F, Nübel U, Witte W, and Werner G. 2014. Insufficient discriminatory power of MALDI-TOF mass spectrometry for typing of Enterococcus faecium and Staphylococcus aureus isolates. Journal of Microbiological Methods 100:5869. 10.1016/j.mimet.2014.02.015

Lu J-J, Tsai F-J, Ho C-M, Liu Y-C, and Chen C-J. 2012. Peptide biomarker discovery for identification of methicillin-resistant and vancomycin-intermediate Staphylococcus aureus strains by MALDI-TOF. Analytical chemistry 84:5685-5692.

O'Hara FP, Suaya JA, Ray GT, Baxter R, Brown ML, Mera RM, Close NM, Thomas E, and AmrineMadsen H. 2016. spa Typing and Multilocus Sequence Typing Show Comparable Performance in a Macroepidemiologic Study of Staphylococcus aureus in the United States. Microb Drug Resist 22:88-96. 10.1089/mdr.2014.0238

Queck SY, Khan BA, Wang R, Bach T-HL, Kretschmer D, Chen L, Kreiswirth BN, Peschel A, DeLeo FR, and Otto M. 2009. Mobile genetic element-encoded cytolysin connects virulence to methicillin resistance in MRSA. PLoS Pathog 5:e1000533.

Recker M, Laabei M, Toleman MS, Reuter S, Saunderson RB, Blane B, Torok ME, Ouadi K, Stevens E, Yokoyama M, Steventon J, Thompson L, Milne G, Bayliss S, Bacon L, Peacock SJ, and Massey RC. 2017. Clonal differences in Staphylococcus aureus bacteraemiaassociated mortality. Nat Microbiol 2:1381-1388. 10.1038/s41564-017-0001-x

Sauget M, Valot B, Bertrand X, and Hocquet D. 2017. Can MALDI-TOF Mass Spectrometry Reasonably Type Bacteria? Trends Microbiol. 10.1016/j.tim.2016.12.006

Schuenck RP, Cavalcante FS, Emery E, Giambiagi-de Marval M, and dos Santos KR. 2012. Staphylococcus aureus isolates belonging to different multilocus sequence types present specific virulence gene profiles. FEMS Immunol Med Microbiol 65:501-504. 10.1111/j.1574-695X.2012.00958.x

Schwarze K, Buchanan J, Taylor JC, and Wordsworth S. 2018. Are whole-exome and wholegenome sequencing approaches cost-effective? A systematic review of the literature. Genet Med. 10.1038/gim.2017.247

Stephens MA. 1974. EDF Statistics for Goodness of Fit and Some Comparisons. Journal of the American Statistical Association 69:730-737. 10.1080/01621459.1974.10480196

Struelens MJ, Hawkey PM, French GL, Witte W, and Tacconelli E. 2009. Laboratory tools and strategies for methicillin-resistant Staphylococcus aureus screening, surveillance and typing: state of the art and unmet needs. Clin Microbiol Infect 15:112-119. 
402

403

404

405

406

407

408

409

410

411

412

413

414

415

416

417

418

419

420

421

422

423

424

425

426

427

428

429

430

431

432

433

434

435

436

10.1111/j.1469-0691.2009.02698.x

Thaipadungpanit J, Amornchai P, Nickerson EK, Wongsuvan G, Wuthiekanun V, Limmathurotsakul D, and Peacock SJ. 2015. Clinical and molecular epidemiology of Staphylococcus argenteus infections in Thailand. J Clin Microbiol 53:1005-1008. 10.1128/JCM.03049-14

Tong SY, Schaumburg F, Ellington MJ, Corander J, Pichon B, Leendertz F, Bentley SD, Parkhill J, Holt DC, Peters G, and Giffard PM. 2015. Novel staphylococcal species that form part of a Staphylococcus aureus-related complex: the non-pigmented Staphylococcus argenteus sp. nov. and the non-human primate-associated Staphylococcus schweitzeri sp. nov. Int J Syst Evol Microbiol 65:15-22. 10.1099/ijs.0.062752-0

Ueda O, Tanaka S, Nagasawa Z, Hanaki H, Shobuike T, and Miyamoto H. 2015. Development of a novel matrix-assisted laser desorption/ionization time-of-flight mass spectrum (MALDITOF-MS)-based typing method to identify meticillin-resistant Staphylococcus aureus clones. J Hosp Infect 90:147-155. 10.1016/j.jhin.2014.11.025

Walter J, Haller S, Blank HP, Eckmanns T, Abu Sin M, and Hermes J. 2015. Incidence of invasive meticillin-resistant Staphylococcus aureus infections in Germany, 2010 to 2014. Euro Surveill 20. 10.2807/1560-7917.ES.2015.20.46.30067

Wang JL, Wang JT, Sheng WH, Chen YC, and Chang SC. 2010. Nosocomial methicillin-resistant Staphylococcus aureus (MRSA) bacteremia in Taiwan: mortality analyses and the impact of vancomycin, MIC = $2 \mathrm{mg} / \mathrm{L}$, by the broth microdilution method. BMC Infect Dis 10:159. 10.1186/1471-2334-10-159

Wang WY, Chiueh TS, Sun JR, Tsao SM, and Lu JJ. 2012. Molecular typing and phenotype characterization of methicillin-resistant Staphylococcus aureus isolates from blood in Taiwan. PLoS One 7:e30394. 10.1371/journal.pone.0030394

Wang WY, Lee SY, Chiueh TS, and Lu JJ. 2009. Molecular and phenotypic characteristics of methicillin-resistant and vancomycin-intermediate staphylococcus aureus isolates from patients with septic arthritis. J Clin Microbiol 47:3617-3623. 10.1128/jcm.00539-09

Wolters M, Rohde H, Maier T, Belmar-Campos C, Franke G, Scherpe S, Aepfelbacher M, and Christner M. 2011. MALDI-TOF MS fingerprinting allows for discrimination of major methicillin-resistant Staphylococcus aureus lineages. Int J Med Microbiol 301:64-68. 10.1016/j.jimm.2010.06.002

Zhang T, Ding J, Rao X, Yu J, Chu M, Ren W, Wang L, and Xue W. 2015. Analysis of methicillinresistant Staphylococcus aureus major clonal lineages by Matrix-Assisted Laser Desorption Ionization-Time of Flight Mass Spectrometry (MALDI-TOF MS). J Microbiol Methods 117:122-127. 10.1016/j.mimet.2015.08.002 
Figure 1

Scatter plot of various MLST types isolates

The peaks at $m / z 3277$ and $m / z 6594$ served as the $x$ and $y$ axes, respectively. The intensities of the characteristic peaks were expressed in arbitrary intensity units. The ellipses represent the $95 \%$ confidence intervals of peak intensities for each MLST type. In the preliminary analysis, the various MLST types could not be satisfactorily separated based only on two characteristic peaks. ST5: red crosses, ST59: green circles, ST239: blue squares, and other ST types: yellow diamonds.

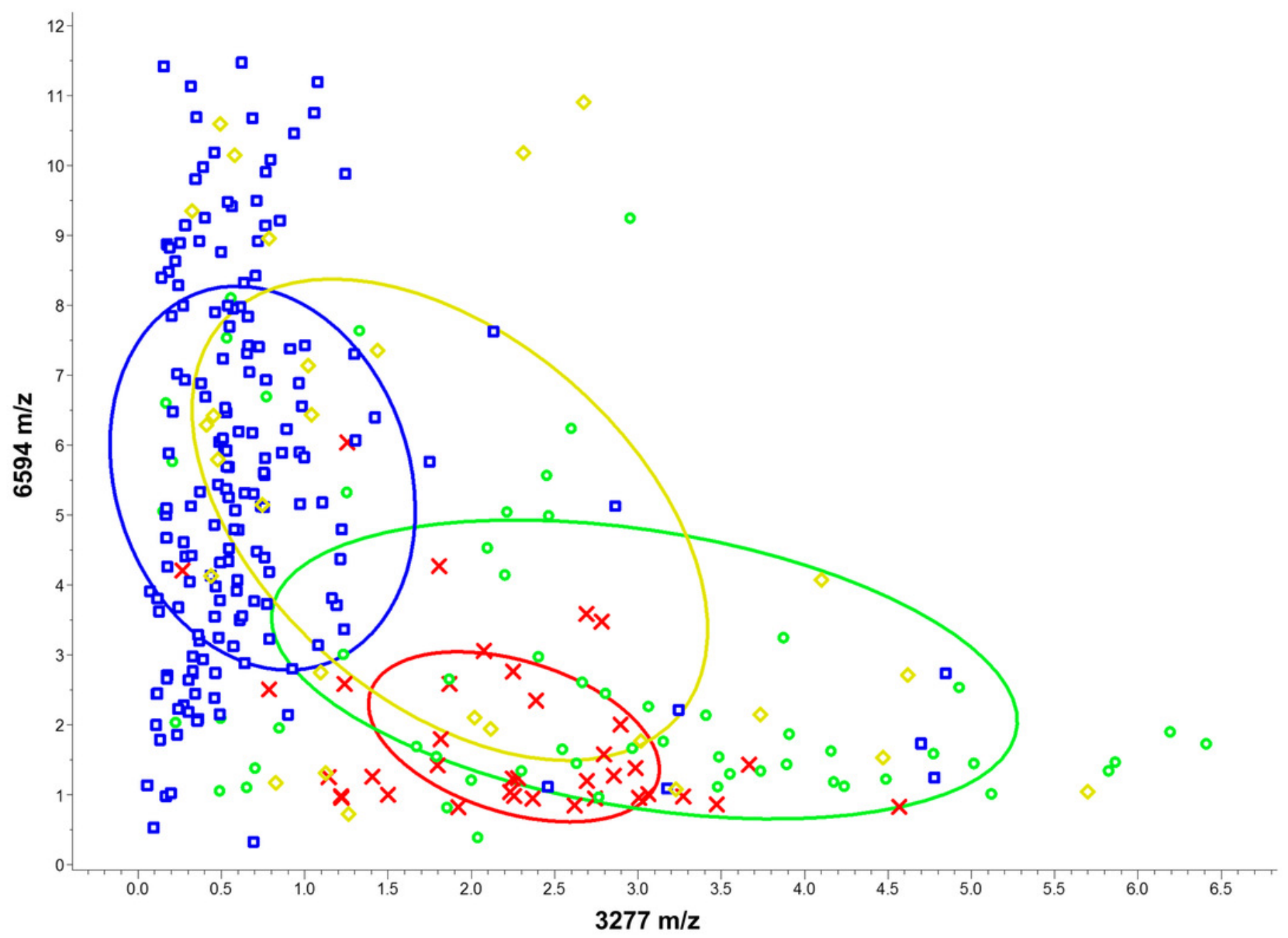


Figure 2

Average spectra of characteristic peaks among various MLST types

Intensities of characteristic peaks ( $m / z$ 2082, 2416, 2430, 2880, 2980, 3277, 3877, 3893, 6553, and 6594, from A to J, respectively) in ST5 (red), ST59 (green), ST239 (blue), and other ST types (yellow) expressed in arbitrary intensity units.

A

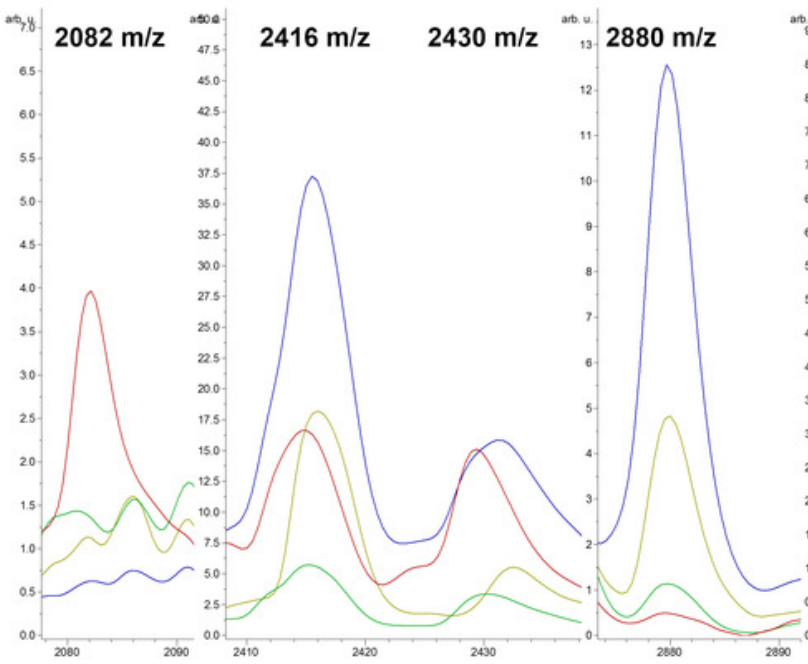

E

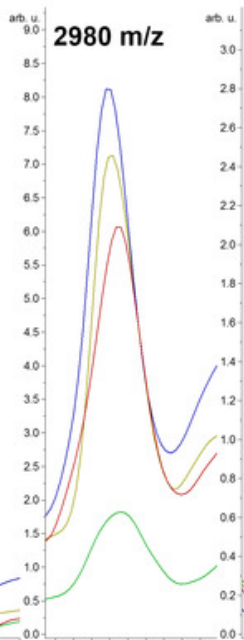

$3277 \mathrm{~m} / \mathrm{z}_{70}^{20.0}$

$3877 \mathrm{~m} / \mathrm{z}$

$3893 \mathrm{~m} / \mathrm{z}$

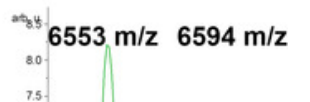

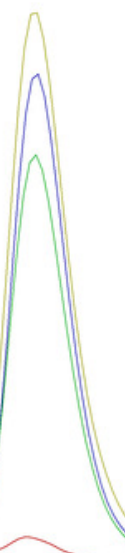
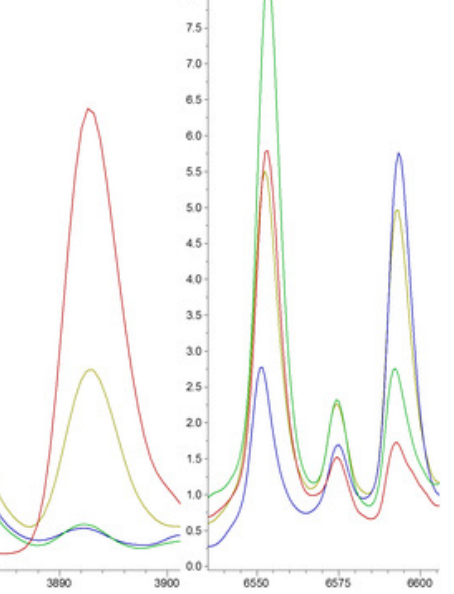
Figure 3

Scatter plot of the ST5 and non-ST5 isolates

The peaks at $m / z 3893$ and $m / z 3877$ served as the $x$ and $y$ axes, respectively. Intensities of the characteristic peaks were expressed in arbitrary intensity units. The ellipses represent the $95 \%$ confidence intervals of peak intensities for ST5 (red ellipse) or non-ST5 (green ellipse). ST5: red crosses, non-ST5: green circles.

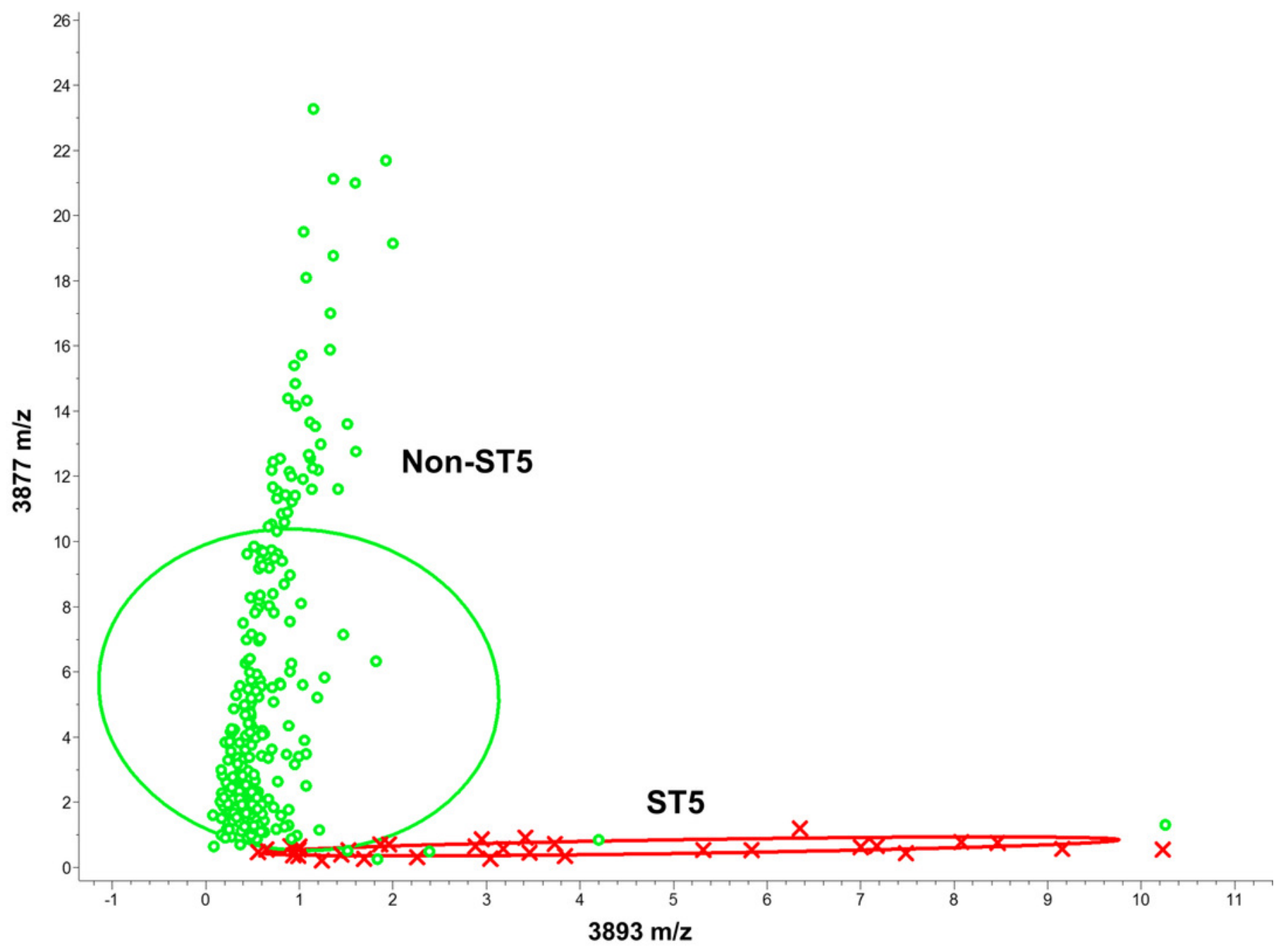




\section{Figure 4}

Scatter plot of the ST59 and non-ST59 isolates

The peaks at $m / z 2416$ and $m / z 2980$ served as the $x$ and y axes, respectively. The intensities of the characteristic peaks were expressed in arbitrary intensity units. The ellipses represent $95 \%$ confidence intervals of the peak intensities for ST59 (red ellipse) or non-ST59 (green ellipse). ST59: red crosses, non-ST59: green circles.

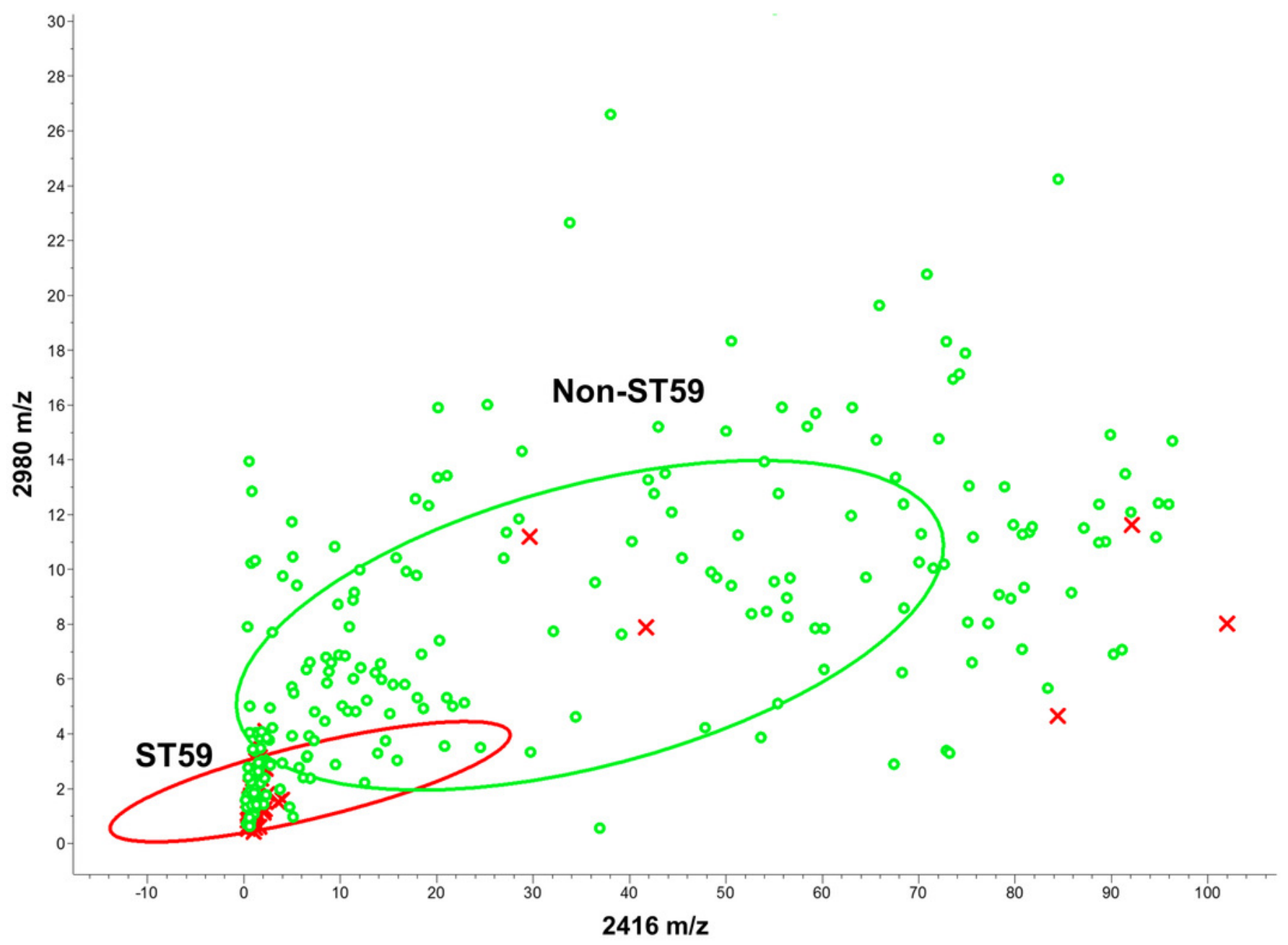


Figure 5

Scatter plot of the ST239 and non-ST239 isolates

The peaks at $m / z 3277$ and $m / z 6553$ served as the $x$ and $y$ axes, respectively. The intensities of the characteristic peaks were expressed in arbitrary intensity units. The ellipses represent 95\% confidence intervals of the peak intensities for ST239 (red ellipse) or nonST239 (green ellipse). ST239: red crosses, non-ST239: green circles.

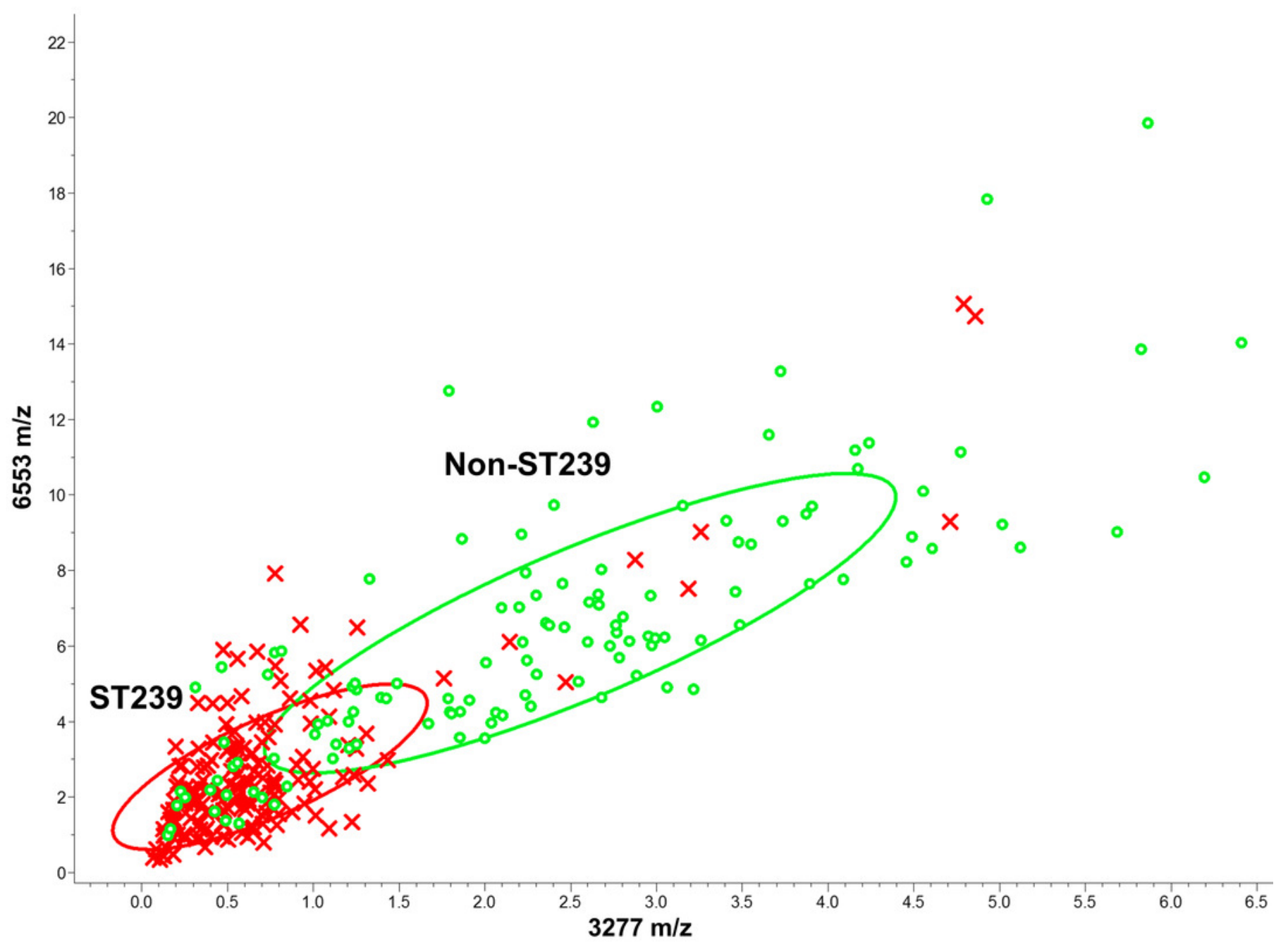




\section{Table $\mathbf{1}$ (on next page)}

Characteristic MALDI-TOF MS peaks in different MLST types of MRSA

Abbreviations: DAve: difference between the maximal and the minimal average peak intensity of all classes; PW/KW: $P$ value obtained through Wilcoxon/Kruskal-Wallis test; PAD: $P$ value obtained through Anderson-Darling test, MALDI-TOF MS: matrix-assisted laser desorption ionization time-of-flight mass spectrometry 
1 Table 1:

2 Characteristic MALDI-TOF MS peaks in different MLST types of MRSA

\begin{tabular}{|c|c|c|c|c|c|c|c|}
\hline \multirow[t]{2}{*}{ Mass } & \multirow[t]{2}{*}{ DAve } & \multirow[t]{2}{*}{$\mathbf{P W} / \mathbf{K W}$} & \multirow[t]{2}{*}{ PAD } & \multicolumn{4}{|c|}{ Average of peak Intensity } \\
\hline & & & & ST5 & ST59 & ST239 & Other ST \\
\hline 2082.13 & 4.28 & $<0.000001$ & $<0.000001$ & 6.13 & 3.84 & 1.85 & 3.24 \\
\hline 2415.79 & 38.19 & $<0.000001$ & $<0.000001$ & 24.57 & 6.13 & 44.32 & 19.72 \\
\hline 2430.49 & 18.13 & $<0.000001$ & $<0.000001$ & 20.12 & 3.86 & 21.99 & 6.23 \\
\hline 2880.03 & 11.2 & $<0.000001$ & $<0.000001$ & 1.15 & 1.88 & 12.35 & 5.38 \\
\hline 2980.45 & 6.85 & $<0.000001$ & $<0.000001$ & 7.4 & 2.47 & 9.32 & 7.88 \\
\hline 3276.8 & 2.94 & $<0.000001$ & $<0.000001$ & 2.61 & 3.73 & 0.79 & 2.08 \\
\hline 3876.96 & 6.24 & $<0.000001$ & $<0.000001$ & 0.75 & 5.5 & 6.33 & 7 \\
\hline 3892.91 & 4.91 & $<0.000001$ & $<0.000001$ & 5.61 & 0.69 & 0.87 & 2.77 \\
\hline 6553.03 & 6.23 & $<0.000001$ & $<0.000001$ & 6.28 & 9.32 & 3.1 & 6.15 \\
\hline 6593.54 & 4.33 & $<0.000001$ & $<0.000001$ & 1.98 & 3 & 6.3 & 5.48 \\
\hline
\end{tabular}

3 Abbreviations: DAve: difference between the maximal and the minimal average peak intensity of 4 all classes; PW/KW: $P$ value obtained through Wilcoxon/Kruskal-Wallis test; PAD: $P$ value 5 obtained through Anderson-Darling test, MALDI-TOF MS: matrix-assisted laser desorption 6 ionization time-of-flight mass spectrometry 


\section{Table 2 (on next page)}

Test performance of various lineage typing ML models

QC, QuickClassifier; SNN, supervised neural network; GA, genetic algorithm; KNN, K-Nearest Neighbor. The number following "GA-KNN" indicates the number of nearest neighbors used in models.

Selected peaks: number of peaks selected by the models; Acc, accuracy; Sen, sensitivity; Spe, specificity. Performance metrics are expressed as mean \pm standard error. 
1 Table 2. Test performance of various lineage typing ML models

\begin{tabular}{|c|l|c|c|c|c|}
\hline ST & Model & $\begin{array}{c}\text { Selected } \\
\text { peaks }\end{array}$ & Acc & Sen & Spe \\
\hline \multirow{5}{*}{ ST5 } & QC & 8 & $0.94 \pm 0.04$ & $0.94 \pm 0.05$ & $0.95 \pm 0.05$ \\
\cline { 2 - 6 } & SNN & 24 & $0.63 \pm 0.05$ & $0.91 \pm 0.05$ & $0.34 \pm 0.04$ \\
\cline { 2 - 6 } & GA_KNN1 & 30 & $0.83 \pm 0.03$ & $0.68 \pm 0.03$ & $0.98 \pm 0.05$ \\
\cline { 2 - 6 } & GA_KNN3 & 30 & $0.84 \pm 0.03$ & $0.71 \pm 0.03$ & $0.97 \pm 0.02$ \\
\cline { 2 - 6 } & GA_KNN5 & 19 & $0.83 \pm 0.03$ & $0.68 \pm 0.03$ & $0.98 \pm 0.02$ \\
\cline { 2 - 6 } & GA_KNN7 & 14 & $0.88 \pm 0.03$ & $0.79 \pm 0.03$ & $0.97 \pm 0.02$ \\
\hline \multirow{5}{*}{ ST59 } & QC & 22 & $0.76 \pm 0.03$ & $0.85 \pm 0.04$ & $0.67 \pm 0.04$ \\
\cline { 2 - 6 } & SNN & 5 & $0.68 \pm 0.04$ & $0.57 \pm 0.05$ & $0.79 \pm 0.04$ \\
\cline { 2 - 6 } & GA_KNN1 & 30 & $0.78 \pm 0.03$ & $0.62 \pm 0.04$ & $0.94 \pm 0.03$ \\
\cline { 2 - 6 } & GA_KNN3 & 29 & $0.82 \pm 0.02$ & $0.70 \pm 0.02$ & $0.94 \pm 0.03$ \\
\cline { 2 - 6 } & GA_KNN5 & 29 & $0.85 \pm 0.03$ & $0.74 \pm 0.03$ & $0.96 \pm 0.02$ \\
\cline { 2 - 6 } & GA_KNN7 & 22 & $0.82 \pm 0.02$ & $0.67 \pm 0.02$ & $0.97 \pm 0.02$ \\
\hline \multirow{5}{*}{ ST239 } & QC & 6 & $0.81 \pm 0.02$ & $0.91 \pm 0.01$ & $0.72 \pm 0.02$ \\
\cline { 2 - 7 } & SNN & 1 & $0.58 \pm 0.03$ & $0.72 \pm 0.03$ & $0.44 \pm 0.04$ \\
\cline { 2 - 7 } & GA_KNN1 & 30 & $0.80 \pm 0.02$ & $0.88 \pm 0.03$ & $0.72 \pm 0.02$ \\
\cline { 2 - 7 } & GA_KNN3 & 30 & $0.80 \pm 0.02$ & $0.85 \pm 0.02$ & $0.74 \pm 0.02$ \\
\cline { 2 - 7 } & GA_KNN5 & 29 & $0.81 \pm 0.02$ & $0.89 \pm 0.01$ & $0.72 \pm 0.02$ \\
& GA_KNN7 & 28 & $0.83 \pm 0.02$ & $0.90 \pm 0.02$ & $0.76 \pm 0.02$ \\
\hline
\end{tabular}

2 QC, QuickClassifier; SNN, supervised neural network; GA, genetic algorithm; KNN, K-Nearest

3 Neighbor. The number following "GA-KNN" indicates the number of nearest neighbors used in 4 models.

5 Selected peaks: number of peaks selected by the models; Acc, accuracy; Sen, sensitivity; Spe, 6 specificity. Performance metrics are expressed as mean \pm standard error. 


\section{Table 3(on next page)}

Accuracy of various multiclass models in classifying different ST lineages

QC, QuickClassifier; SNN, supervised neural network; GA, genetic algorithm; KNN, K-Nearest Neighbor, the number following "GA-KNN" indicated the number of nearest neighbor used in models; Selected peaks: number of peaks selected by the models. Accuracies were expressed as mean \pm standard error. 
1 Table 3. Accuracy of various multiclass models in classifying different ST lineages.

\begin{tabular}{|l|c|c|c|c|c|}
\hline \multicolumn{1}{|c|}{ Model } & $\begin{array}{c}\text { Selected } \\
\text { peaks }\end{array}$ & ST5 & ST59 & ST239 & Others \\
\hline QC & 24 & $0.65 \pm 0.05$ & $0.85 \pm 0.03$ & $0.79 \pm 0.03$ & $0.09 \pm 0.07$ \\
\hline SNN & 25 & $0.65 \pm 0.05$ & $0.34 \pm 0.05$ & $0.25 \pm 0.06$ & $0.13 \pm 0.09$ \\
\hline GA_KNN1 & 30 & $0.58 \pm 0.05$ & $0.63 \pm 0.04$ & $0.84 \pm 0.02$ & $0.09 \pm 0.07$ \\
\hline GA_KNN3 & 26 & $0.73 \pm 0.03$ & $0.78 \pm 0.03$ & $0.90 \pm 0.02$ & $0.13 \pm 0.08$ \\
\hline GA_KNN5 & 27 & $0.73 \pm 0.03$ & $0.78 \pm 0.03$ & $0.94 \pm 0.02$ & $0.04 \pm 0.07$ \\
\hline GA_KNN7 & 22 & $0.92 \pm 0.03$ & $0.81 \pm 0.03$ & $0.94 \pm 0.02$ & $0.04 \pm 0.07$ \\
\hline
\end{tabular}

2 QC, QuickClassifier; SNN, supervised neural network; GA, genetic algorithm; KNN, K-Nearest

3 Neighbor, the number following "GA-KNN" indicated the number of nearest neighbor used in

4 models; Selected peaks: number of peaks selected by the models. Accuracies were expressed as

5 mean \pm standard error. 